\title{
Gestaltungsmuster des Mathematikunterrichts \\ in Jahrgangs- und \\ Mehrjahrgangsklassen - \\ Zwei Kantone im Vergleich
}

\section{Esther Brunner und Andreas Imhof}

In der vorliegenden Studie wurde in den Kantonen Thurgau und Graubünden geprüft, inwiefern sich der selbstberichtete Einsatz bestimmter Unterrichts- und Sozialformen im Mathematikunterricht in Jahrgangs- und Mehrjahrgangsklassen der Primarschule unterscheidet. Die Befragung wurde bei erfahrenen Lehrpersonen und bei solchen, die am Beginn ihrer professionellen Laufbahn stehen, durchgeführt. Der Datensatz lässt Vergleiche zwischen den Kantonen, zwischen Experten und Novizen sowie zwischen Jahrgangs- und Mehrjahrgangsklassen zu. Die Ergebnisse zeigen, dass sich bezüglich selbstberichteter Gestaltung von Mathematikunterricht weit mehr Gemeinsamkeiten als Unterschiede zeigen. Zudem konnten sechs Faktoren extrahiert werden, die als unterschiedliche Gestaltungsprofile interpretiert werden können.

\section{Einleitung}

Mehrjahrgangsklassen sind in ländlichen Gebieten der Schweiz wie auch in Süddeutschland und in Vorarlberg als kleine Dorfschulen verbreitet (Raggl, Smit \& Kerle, 2015). Dies ist auch in den Kantonen Thurgau und Graubünden der Fall, in denen Mehrjahrgangsklassen eine langjährige Tradition haben (Trachsler, 2008). Vergleichsweise neu ist die Schaffung von Mehrjahrgangsklassen ohne strukturelle Notwendigkeit ${ }^{1}$ aus pädagogischen Überlegungen. Mehrjahrgangsklassen werden dabei als eine besonders geeignete Schulform interpretiert, die einen konstruktiven Umgang mit der Heterogenität der Schülerschaft mit sich bringe, da vermehrt erweiterte Lehr- und Lernformen eingesetzt würden und die Qualität des Unterrichts durch eine grössere Binnendifferenzierung gesteigert werde:

Die Verschiedenheit der Kinder soll nicht nur angenommen, sondern bewusst verstärkt und genutzt werden für ein Mit- und Voneinander-Lernen und zur Förderung der sozialen Kompetenzen. Die Pädagogik des jahrgangsübergreifenden Lernens kann somit als ein Ansatz in der Realisierung einer Pädagogik der Vielfalt betrachtet werden. (Monn, 2011, S. 9) 
Diesen Überlegungen folgend wurden Jahrgangsklassen der Primarschule in einigen Schweizer Kantonen, wie z.B. im Thurgau ${ }^{2}$, zugunsten von von Mehrjahrgangsklassen ${ }^{3}$ und jahrgangsübergreifendem Lernen aufgehoben. Mittlerweile weist der Kanton Thurgau in den Primarschulen einen der höchsten Anteile von Mehrjahrgangsklassen schweizweit auf (Monn, 2011). Dabei geht es weniger um die Einführung von Mehrjahrgangsklassen an sich, als um die Implementierung einer bestimmten Schulkultur durch die Schaffung von Mehrjahrgangsklassen, die als «vielversprechend» (Trachsler, 2008, S. 1) bezeichnet werden.

Im ländlich-alpinen Raum, so auch im Kanton Graubünden, ist der altersgemischte Unterricht nicht eine pädagogisch begründete Unterrichtsmassnahme, sondern «eine notwendige organisatorische Massnahme [...], um kleine Schulen auch bei geringer SchülerInnenzahl erhalten zu können» (Raggl, 2011, S. 237). Die Führung von Mehrjahrgangsklassen ist wenig umstritten, da oft nur dadurch die Dorfschule erhalten werden kann.

Unabhängig der Gründe, die zur Schaffung von Mehrjahrgangsklassen führen, zeigen verschiedene Studien, dass sich Lehrpersonen aus Mehrjahrgangsklassen von der zusätzlichen Heterogenität stark belastet fühlen (z. B. Mulryan-Kyne, 2004; Raggl, 2011) und mehrheitlich jahrgangsgetrennt arbeiten (Brunner, 2017; Hyry-Beihammer \& Hascher, 2015; Rossbach, 2003). Es ist deshalb offen, inwiefern es gelingt, durch die Schaffung von Mehrjahrgangsklassen vermehrt Formen von Binnendifferenzierung zu implementieren.

Dies wird im Rahmen der Studie für den Mathematikunterricht genauer untersucht. Gefragt wird erstens nach selbstberichteten Gestaltungsunterschieden von Mathematikunterricht von Primarlehrpersonen, die in Jahrgangsbzw. Mehrjahrgangsklassen unterrichten. Zweitens interessiert, inwiefern sich allfällige Gestaltungsunterschiede zwischen erfahrenen Lehrpersonen und solchen, die am Anfang ihrer Berufskarriere stehen, zeigen. Und drittens fragt der Beitrag nach Unterschieden und Gemeinsamkeiten der Gestaltungsmuster für zwei Kantone, die beide eine langjährige Tradition mit Mehrjahrgangsklassen haben, sich aber in der Bewertung der (freiwilligen) Altersdurchmischung unterscheiden.

\section{Theoretische Grundlagen}

\section{Schulstruktur und Altersheterogenität}

Die Schulstruktur Jahrgangs- bzw. Mehrjahrgangsklasse schafft den Rahmen für die Organisation der individuellen und gemeinsamen Lernprozesse. Obwohl eine Jahrgangsklasse auch altersheterogen ist, beschränkt sich die Heterogenität aber in der Regel auf maximal drei Entwicklungsjahrgänge (Largo, 2013). Eine Mehrjahrgangsklasse hingegen umfasst eine deutlich grössere Altersspanne, je nachdem wie viele Jahrgänge zusammengefasst werden. Dass es bei einer grösseren Altersheterogenität automatisch zu einer grösseren Binnendifferen- 
zierung kommt, ist nicht zwingend. Dennoch werden im Zusammenhang mit Mehrjahrgangsklassen «Individualisierung und Altersmischung als Teil einer neuen Lernkultur» (Thurn, 2010, S. 29) interpretiert. Begründet wird dies durch die Notwendigkeit der Differenzierung, die als «Voraussetzung für das Lernen in jahrgangsübergreifenden Gruppen» (Kucharz \& Wagener, 2009, S. 11) betrachtet wird. Der Umkehrschluss, über Altersdurchmischung eine verstärkte Differenzierung zu erreichen, ist trotz fehlender Korrektheit verbreitet (z. B. Laging, 2003), und die Erwartungen sind entsprechend hoch: "Jahrgangsübergreifendes Lernen ist [...] die pädagogisch richtige Antwort, nicht nur für soziales Lernen [...] sondern vor allem für fachliches» (Thurn, 2010, S. 29). Als kontrastierende Denkfigur wird argumentativ die Jahrgangsklasse herangezogen und entsprechend problematisiert:

Die Jahrgangsklasse ist eine verwaltungstechnische Einheit [...] Mit der Einführung von altersgemischten Gruppen entfällt die Gleichschrittigkeit und Vereinheitlichung des Lernens (Laging, 2003, S. 20)

Trotz der grundsätzlichen Problematisierung der Jahrgangsklasse ist der jahrgangsbezogene Abteilungsunterricht ${ }^{4}$ auch in Mehrjahrgangsklassen nebst weiteren Organisationsformen altersdurchmischten Lernens ${ }^{5}$ - verbreitet (Brunner, 2017; Hyry-Beihammer \& Hascher, 2015; Rossbach, 2003).

Insgesamt liegen nur wenige Forschungsbefunde vor, die Aussagen darüber erlauben, wie die Schulstruktur der Mehrjahrgangsklasse wirkt. Die vorhandenen Ergebnisse beziehen sich vorwiegend auf die Leistungen in Mathematik und Erstsprache sowie auf die sozialen Fähigkeiten (z. B. Kadivar, Navabi Nejad \& Madadi Emamzade, 2005; Rossbach, 2003). Hattie (2009, S. 92) bescheinigt der Altersdurchmischung einen sehr geringen Einfluss auf Leistung oder Kooperation und geht von einem vernachlässigbaren Effekt $(d=.04)$ aus. Grittner, Hartinger und Rehle (2013) belegen überdies, dass jahrgangsgemischter Unterricht insbesondere für die jüngeren Lernenden in Mathematik Vorteile mit sich bringt, nicht aber für die älteren.

Auf der anderen Seite ist es evident, dass Mehrjahrgangsklassen für die Lehrpersonen mit einer höheren Komplexität des Unterrichtsgeschehens und der Unterrichtsplanung einhergehen. Wird in Mehrjahrgangsklassen entlang des jahrgangsbezogenen Abteilungsunterrichts gearbeitet, bedeutet dies für die Lehrpersonen, dass sie parallel mit den einzelnen Klassen unterschiedliche Themen bearbeiten und vorbereiten müssen. Arbeiten die Lehrpersonen nach dem Konzept des «Lernens am gemeinsamen Gegenstand» (Feuser, 2013) setzt dies voraus, dass sie niveaudifferenzierende Angebote für Lernende unterschiedlichen Alters gestalten können. Dass dies als Belastung empfunden werden kann, zeigen verschiedene Studien (z. B. Brunner, 2017; Mulryan-Kyne, 2004), ebenso wie auch die Angst vor Überforderung oder davor, den Überblick zu verlieren (Wagener, 2014a).

Befunde dazu, wie Lehrpersonen in Mehrjahrgangsklassen ihren Unterricht gestalten und ob sie tatsächlich mehr erweiterte Lehr-/Lernformen oder 
Methoden offenen Unterrichts (Abschnitt 2.2) einsetzen als Lehrpersonen in Jahrgangsklassen, fehlen weitgehend. Carle und Berthold (2004) konnten aber eine höhere Differenzierung im Unterricht bei Lehrpersonen nachweisen, die altersdurchmischte Klassen bevorzugen (Wagener, 2014b). Qualitativ angelegte Studien ermöglichen Einblicke in mathematische Gesprächsführung und den Wissensaufbau in altersdurchmischten Gruppen (z. B. Nührenbörger, 2009; Schülke, 2013), aber Auswirkungen der Schulstruktur auf die Unterrichtsgestaltung in den einzelnen Fächern werden vergleichsweise wenig diskutiert, obwohl verschiedene didaktische Konzepte und Strategien für den Unterricht mit altersdurchmischten Klassen angeboten werden (z. B. Zumwald, 2012) oder für den Mathematikunterricht an einzelnen Themen konkretisiert vorliegen (z. B. Hengartner et al., 2010; Nührenbörger \& Pust, 2006; Rathgeb-Schnierer $\&$ Rechtsteiner-Merz, 2010).

Während sich altersdurchmischtes Lernen in Inhaltsbereichen, in denen das Curriculum vergleichsweise offen ist, relativ gut umsetzen lässt, zeigen sich für den hierarchischen Stoffaufbau in Mathematik deutliche Herausforderungen, weil die Inhalte nicht in einer beliebigen Reihenfolge bearbeitet werden können, sondern systematisch aufeinander bezogen werden müssen. Es ergibt deshalb Sinn, gerade diesen Fachbereich zu fokussieren und den Einsatz unterschiedlicher Unterrichts- und Sozialformen zu untersuchen.

\section{Unterricht als Möglichkeitsraum}

Im Sinne eines Angebot-Nutzungs-Modells von Unterricht (Fend, 1998; Reusser \& Pauli, 2010) stellt der Unterricht einen Möglichkeitsraum für das Lernen dar. Bestimmt wird das Angebot durch strukturelle Rahmenbedingungen, durch Merkmale der Lehrpersonen und Voraussetzungen der Lernenden. Auf der unmittelbar sichtbaren Ebene des Unterrichts, der sogenannten Oberflächenstruktur (Reusser, 2005), wird das Angebot deutlich in verschiedenen Unterrichts- und Sozialformen.

Unabhängig davon, welche Schulstruktur (Jahrgangs- oder Mehrjahrgangsklasse) vorherrscht, gelten Qualitätsansprüche an das Bildungsangebot. Empirisch liessen sich verschiedene Merkmale von Unterricht bestimmen, welche die Leistungsentwicklung der Lernenden positiv beeinflussen, z. B. Aspekte wie Klarheit, Motivierungsqualität, Klassenführung oder die Nutzung der effektiven Lernzeit (z. B. Brophy \& Good, 1986). Darüber hinaus werden Dimensionen wie Schülerorientierung, Differenzierung und Umgang mit Heterogenität diskutiert (Helmke, 2012). Aus motivationalen Gründen wird zudem die Bedeutung der Methodenvielfalt hervorgehoben (Helmke, 2012). Eine vielfältige Angebotsgestaltung betrifft verschiedene Dimensionen, z. B. die Steuerung durch die Lernenden oder die Möglichkeiten zum sozialen Austausch. Innerhalb der beiden Dimensionen Steuerung und Sozialform können verschiedene Unterrichtsformen angesiedelt werden. Wie in Abbildung 1 dargestellt, wird z. B. Planarbeit als Einzelarbeit mit wenig Steuerung durch 
die Lernenden verortet, während Projektarbeit als gruppenbezogenes Lernen mit grosser Steuerung durch die Lernenden eingeschätzt wird (Bildungsdirektion Kanton Zürich, 2007).

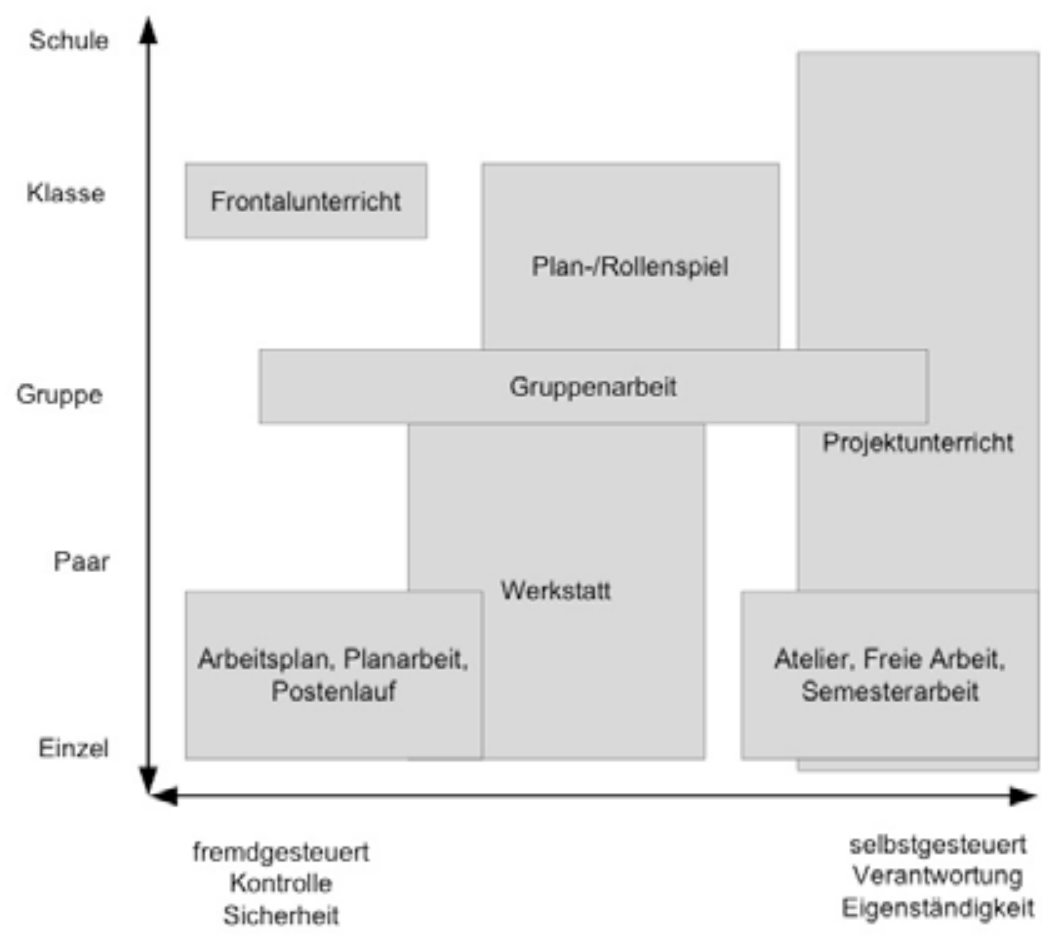

Abbildung 1: Unterrichtsformen innerhalb der Dimensionen Steuerung und Sozialform (Bildungsdirektion Kanton Zürich, 2007, S. 5).

Diese Unterrichtsformen lassen sich durch weitere Lehr- und Lernformen ergänzen und darüber hinaus gruppieren. Frontalunterricht wird einem eher traditionell geprägten Gestaltungsmuster zugeschrieben, zu dem auch Formen wie Erklären, Vorzeigen, Abfragen und Vorrechnen lassen, gehören (Rakoczy, Buff \& Lipowsky, 2005). Rakoczy et al. (2005) sprechen in Abgrenzung zum traditionellen Unterricht von «offenen Lernformen und innovativem Unterricht». Eher als innovative Formen werden Werkstatt-, Projektunterricht und Freie Arbeit bezeichnet, die eine grössere Steuerung bei den Lernenden vorsehen, wobei die Bezeichnung «innovativ» ungünstig ist, da sie eine Wertung impliziert statt einer Deskription. Arbeitspläne, Planarbeit und Postenlauf stellen weniger eine innovative Lehr-/Lernform dar, als dass sie für die Bearbeitung der meist wenig offenen Aufgabenstellungen eine gewisse Wahlfreiheit bezüglich inhaltlicher und zeitlicher Bearbeitung zulassen, entweder in der Reihenfolge der 
Bearbeitung oder aber in der zumindest gelegentlichen Wahl der Sozialform. Die Offenheit bezieht sich also primär auf die Situation, nicht auf den Inhalt. Die Verortung in der Abbildung ist zwar bezüglich Steuerung zutreffend, hinsichtlich der Sozialform lassen diese Formen aber mehr Freiräume zu. Als weitere relevante Dimension des Unterrichts gilt aus ko-konstruktivistischer Perspektive (z. B. Greeno, 2006) die Möglichkeit des Diskurses und fachlichen Austausches. Gruppiert man Lehr- und Lernformen entlang dieser Dimension, lassen sich Formen wie das Unterrichtsgespräch, die Präsentation oder Diskussion von Arbeiten durch die Lernenden nennen. Inhaltlich und prozedural unbestimmt sind Formen, die ausschliesslich entlang der Sozialform definiert werden wie Gruppen- oder Partnerarbeit.

Unterrichtsformen bilden den Rahmen für die Organisation von geeigneten Lehr-Lern-Arrangements. Je nach inhaltlicher Zielsetzung ist die eine oder andere Unterrichtsform geeigneter. Stehen die Förderung von mathematischen Argumentations- und Begründungskompetenzen im Mittelpunkt, sind Formen geeignet, die einen Diskurs ermöglichen. Wird hingegen ein erlernter Inhalt geübt, ist dies in individueller Einzelarbeit (z. B. im Rahmen eines Arbeitsplans oder einer Werkstatt) gut möglich. Vielfalt in den Unterrichtsformen bedeutet deshalb auch ein Angebot zum Erwerb vielfältiger fachlicher Kompetenzen.

Bezieht man die in Abschnitt 1 erwähnten Erwartungen an die Mehrjahrgangsklasse auf die hier gruppierten Lehr-/Lernformen, lassen sich die Vermutungen präzisieren: Von Mehrjahrgangsklassen wird erwartet, dass sie wegen der Betonung der erweiterten Gelegenheiten zum sozialen Lernen vermehrt Gruppen- und Tandemarbeit sowie mehr diskursive Formen einsetzen, als dies in Jahrgangsklassen der Fall sein dürfte. Die durch die Mehrjahrgangsklasse zu erreichende höhere Binnendifferenzierung müsste sich in der Betonung von innovativen Unterrichtsformen mit einer grösseren Steuerung durch die Lernenden sowie mehr offene Lernformen und weniger Formen traditionellen Unterrichts zeigen.

Da im Kanton Thurgau im Gegensatz zum Kanton Graubünden zahlreiche Mehrjahrgangsklassen ohne strukturelle Notwendigkeit aus pädagogischen Überlegungen gebildet wurden, wäre ein Kantonsunterschied zu vermuten, sofern das Motiv zur Schaffung der Mehrjahrgangsklasse tatsächlich entscheidend sein sollte, wie dies verschiedene Autoren (z. B. Laging, 2003) nahelegen.

\section{Fragestellungen und Hypothesen}

Am Beispiel von je zwei Extremgruppen aus beiden Kantonen - Lehrpersonen in ihren ersten beiden Berufsjahren (Novizinnen/Novizen) und erfahrenen Praxislehrpersonen, die im Rahmen der Ausbildung im Praxisfeld Studierende betreuen (Expertinnen/Experten) - interessieren folgende Fragestellungen:

F1) Unterscheidet sich die selbstberichtete Gestaltung des Mathematikunterrichts bei Primarlehrpersonen in Mehrjahrgangsklassen von derjenigen in Jahrgangsklassen? 
F2) Lassen sich Unterschiede in der selbstberichteten Gestaltung des Mathematikunterrichts bei erfahrenen Praxislehrpersonen und Novizinnen feststellen?

F3) Ist die selbstberichtete Gestaltung des Mathematikunterrichts in den Kantonen Graubünden und Thurgau vergleichbar?

Zur Beantwortung der Fragestellungen wird zunächst getestet, ob sich die selbstberichtete Unterrichtspraxis in Mathematik in einheitliche übergeordnete Gruppen von Unterrichtsformen einteilen lässt (Abschnitt 2.2). Danach werden die zuvor bestimmten übergeordneten Unterrichtsformen auf Unterschiede zwischen den Kantonen, der Schulstruktur und des Expertenstatus hin überprüft. Aufgrund der explorativen Art der Untersuchung, dem Fehlen von Vergleichsdaten und des deskriptiven Zugangs (Befragung) wird jeweils die Nullhypothese getestet.

\section{Methoden}

\section{Stichprobe und Kontext der Studie}

Im Rahmen des Forschungsprojekts «AdL Math» der Pädagogischen Hochschule Thurgau wurden Primarlehrerpersonen zur Gestaltung ihres Mathematikunterrichts befragt. Diese Erhebung wurde analog im Kanton Graubünden durchgeführt. In beiden Kantonen wurden sowohl Primarlehrpersonen einbezogen, die am Anfang ihrer beruflichen Laufbahn stehen (Novizengruppe) sowie erfahrene Lehrpersonen (Expertengruppe), die als Praxislehrpersonen in ihren Klassen Lehramtsstudierende begleiten (Tabelle 1).

Tabelle 1: Stichprobe

\begin{tabular}{lccc}
\hline & $\begin{array}{c}\text { Stichprobe } \\
\text { Thurgau }\end{array}$ & $\begin{array}{c}\text { Stichprobe } \\
\text { Graubünden }\end{array}$ & Total \\
\hline Lehrpersonen Jahrgangsklassen & 52 & 43 & 95 \\
\hline Lehrpersonen Mehrjahrgangsklasse & 99 & 23 & 122 \\
\hline Total & 151 & 66 & 217 \\
\hline Praxislehrpersonen (Experten/Expertinnen) & 50 & 31 & 81 \\
\hline Berufseinsteigende (Novizen/Novizinnen) & 101 & 35 & 136 \\
\hline Total & 151 & 66 & 217 \\
\hline
\end{tabular}

Die Gesamtstichprobe Thurgau umfasst 151 Primarlehrpersonen (Details Tabelle 1). Bei der «Novizengruppe» $(N=101)$, handelt es sich um eine Vollerhebung aller Primarlehrpersonen, die im Schuljahr 2013/2014 in ihrem ersten bzw. zweiten Berufsjahr im Kanton Thurgau unterrichteten. 33 dieser Lehrper- 
sonen $(32.7 \%)$ unterrichten in einer Jahrgangsklasse und 68 (67.3\%) in einer Mehrjahrgangsklasse. Zur Expertengruppe gehören 50 Praxislehrpersonen, die regelmässig Studierende in zwei unterschiedlichen Praktika begleiten (erstes und letztes Praktikum innerhalb der Ausbildung zur Primarlehrperson). Diese Lehrpersonen wurden anlässlich eines obligatorischen Treffens im Herbst 2014 schriftlich mit einem Fragebogen befragt. Lehrpersonen, die in beiden Praktika arbeiten, nahmen an der Befragung nur einmal teil. 19 der Praxislehrpersonen $(38 \%)$ unterrichten in Jahrgangsklassen, 31 (62\%) in Mehrjahrgangsklassen.

Aus organisatorischen Gründen konnte im Kanton Graubünden keine Vollerhebung durchgeführt werden. Es wurde deshalb aufgrund der Daten des Amts für Volksschule des Kantons Graubünden eine Zufallsstichprobe gezogen. Von total 805 Lehrpersonen wurden zufällig 150 Lehrpersonen ausgewählt, die um die Teilnahme an einer Onlineumfrage gebeten wurden. In einem zweiten Schritt wurden aus dem Datensatz alle Lehrpersonen mit den Diplomjahren 2012/13 und 14 zusätzlich angeschrieben $(N=72)^{6}$. Diese zweite Stichprobe wurde gebildet, da im Rahmen einer Zufallsstichprobe nicht mit genügend Berufseinsteigerinnen und Berufseinsteigern für einen Vergleich zum Kanton Thurgau gerechnet werden konnte. Von den 222 angeschriebenen Lehrpersonen konnten 221 brieflich erreicht werden und 99 haben an der Umfrage teilgenommen (Rücklauf $44.8 \%$ ). Von den 99 Lehrpersonen wurden 11 aus der Auswertung ausgeschlossen, da sie entweder keine Mathematik oder lediglich in Kleinpensen unterrichten. Es verbleiben 88 Lehrpersonen (Details Tabelle 1). Davon sind 35 der Novizengruppe zugeordnet, 31 den Praxislehrpersonen und 23 Lehrpersonen mit mehr als 3 Jahren Berufspraxis, aber keinem Mandat als Praxislehrperson. Von den beiden letzten Gruppen wurde für die Analysen aufgrund der Vergleichbarkeit mit dem Kanton TG nur die Praxislehrpersonen berücksichtigt. Bei der Novizengruppe unterrichten 20 der 35 Lehrpersonen $(57 \%)$ in einer Jahrgangsklasse und $15(23 \%)$ in einer Mehrjahrgangsklasse. Bei der Expertengruppe unterrichten 23 der 31 Lehrpersonen (74\%) in einer Jahrgangsklasse und 8 (26\%) in einer Mehrjahrgangsklasse.

Die Gesamtstichprobe weist somit 217 Lehrpersonen auf. Davon unterrichten 122 in Mehrjahrgangsklassen, 136 von ihnen gehören zur Gruppe der Berufseinsteigenden.

\section{Art der Studie und Instrument}

In der Thurgauer Forschungsgruppe wurde ein Instrument entwickelt, das maximal 83 Variablen pro Lehrperson erfasst und das für die Befragung im Kanton Graubünden leicht angepasst wurde. Es handelt sich um einen stark strukturierten Fragebogen (Atteslander, 2003) mit meist geschlossenen Frageformaten des Selektionstyps, der sich auf fünf verschiedene inhaltliche Teile bezieht: 
1) Angaben zur Person

2) Rahmenbedingungen

3) Deskription Mathematikunterricht bezüglich a) Unterrichtsformen,

b) Sozialformen und c) eingesetzten Materials

4) Zusätzliche Deskription von Unterrichtsphasen in Mehrjahrgangsklassen

5) Überzeugungen zum altersdurchmischten Lernen

Die für den vorliegenden Beitrag relevanten Bereiche $3 \mathrm{a}$ und $3 \mathrm{~b}$ werden nachfolgend genauer vorgestellt.

Um die eingesetzten Unterrichtsformen erfassen zu können, wurden 14 Items angeboten, die je eine Lehr-/Lernform beschreiben. Dabei wurde für 13 Items auf eine unveröffentlichte Projektunterlage aus einer schweizerischen Videostudie zu Mathematikunterricht (Pauli \& Reusser, 2001) zurückgegriffen, um bei der Erhebung von Unterrichtsformen möglichst die lokalen Gegebenheiten berücksichtigen zu können. Die Items wurden sprachlich angepasst und für die aktuelle Studie adaptiert. Ein Item (Einsatz von Lernjournal, forschendem Lernen bzw. Reisetagebuch) wurde zusätzlich entwickelt, weil diese Form (Gallin, 2015) im Zusammenhang mit Mehrjahrgangsklassen vorgeschlagen wird (Duss \& Gallin, 2015). Die 14 angebotenen Unterrichtsformen waren: Vortrag/Erklärung, Vorzeigen, Abfragen, Vorrechnen lassen, Lehrgespräch, Diskussionen, Präsentationen der Lernenden, Stillarbeit, Postenarbeit, Werkstattunterricht, Planarbeit, Freiarbeit, Projektunterricht und Forschendes Lernen/Lerntagebuch. Die Lehrpersonen wurden aufgefordert, die durchschnittliche Häufigkeit, mit der sie eine bestimmte Unterrichtsform einsetzen, anzugeben (1: weniger als einmal im Monat; 2: ein- bis zweimal pro Monat; 3: einmal pro Woche; 4: zwei- bis dreimal pro Woche; 5: (fast) jede Lektion). Die fünfstufige Likertskala wurde grosszügig metrisch interpretiert.

Die Häufigkeit der eingesetzten Sozialformen (Klassen-, Gruppen-, Tandem-, Einzelarbeit, Wahl der Sozialform) wurde auf derselben fünfstufigen Likertskala erfasst. Diese fünf Items stammen aus der Projektunterlage von Pauli und Reusser (2001) und wurden nur geringfügig adaptiert und umformuliert.

\section{Datenanalyse}

Zur Komplexitätsreduktion wurden die befragten Unterrichts- und Sozialformen einer Hauptkomponenten-Faktorenanalyse mit Varimax Rotation unterzogen. Die gemeinsame Analyse von Unterrichts- und Sozialformen ist methodisch nicht unproblematisch, da gewisse Unterrichtsformen bestimmte Sozialformen implizieren. Entsprechend könnte die Analyse aufgrund von Multikollinearität verzerrt sein. Durchgeführt wurden deshalb sowohl kombinierte wie getrennte Analysen. Dargestellt werden die Ergebnisse der kombinierten Analyse, einzelne Resultate der getrennten Analyse werden aber zur Ergebnisdarstellung herangezogen. Die kombinierte Analyse wird aus folgenden Gründen bevorzugt: 1) Gestaltungsmuster von Mathematikunterricht werden sowohl durch Unter- 
richts- wie Sozialformen bestimmt. 2) Eine trennscharfe Beurteilung der Häufigkeit der verwendeten Unterrichts- und Sozialformen ist komplex, da der konkrete Unterricht und die Beschreibung nicht vollständig korrespondieren müssen. Es ist deshalb eine summierte Globalangabe von den Lehrpersonen zu erwarten. Einem solchen Globalurteil wird durch eine gemeinsame Analyse eher entsprochen als durch eine getrennte. 3) Die statistischen Werte sprechen gegen das Vorhandensein von Multikollinearität bzw. Singularität in den Daten (keine extrem hohen Korrelationen, bzw. Determinante, vgl. Abschnitt 4.1).

Da die einzelnen Items lediglich quasi-metrisch interpretiert werden können und nicht von symmetrisch wahrgenommen Distanzen ausgegangen werden kann, wurde die Faktorenstruktur zusätzlich mit nichtparametrischen Methoden validiert (nMDS).

Die resultierende Faktorlösung impliziert aufgrund der Inspektion des Screeplots eine zweifaktorielle Lösung7, aufgrund des Kaiser Kriteriums eine sechsfaktorielle Lösung. Beide Lösungen sind theoretisch plausibel begründbar. Wegen der akzeptablen Stichprobengrösse $(N=196)^{8}$, der geringen Anzahl an Items, der resultierenden Kommunalitäten (Durchschnitt $=0.589$ ) und der guten theoretischen Einordnungsmöglichkeit ${ }^{9}$ wurde die Faktorenextraktion nach Kaiser-Kriterium vorgenommen.

Die einzelnen Items wurden bei einer Faktorladung $>0.4$ auf mindestens einem Faktor in die Berechnung der Skalen aufgenommen. Bei mehreren Ladungen über dem Schwellenwert wurde das Item demjenigen Faktor zugeordnet, auf dem dieses am stärksten lädt. Die so gebildeten Skalen werden in der Folge bezüglich signifikanten Haupteffekten und Interaktionen durch eine dreifaktorielle ANOVA bezüglich des Expertenstatus (Novizen und Experten), der Schulstruktur (MJ- und JG-Klassen) sowie auf Kantonsunterschiede (Thurgau und Graubünden) hin überprüft. Da eine Befragung und kein experimentelles Design vorliegt, die Fragestellungen explorativer Art und die gebildeten Skalen konzeptuell unabhängig sind, werden aufgrund der Empfehlungen von Huberty und Morris (1989) einzelne Faktoranalysen einem mehrfaktoriellen Vergleich vorgezogen. Da durch dieses Vorgehen das Problem der Kumulierung des Alphafehlers entsteht, werden signifikante Effekte vor allem aufgrund der Effektstärke interpretiert. Auf Empfehlung von Field (2014) wird die Effektstärke durch das Omega-Quadrat $\left(\omega^{2}\right)$ bestimmt.

\section{Resultate}

Im Folgenden werden die Ergebnisse zu den drei in Abschnitt 2.3 aufgeführten Forschungsfragen dargestellt. Zunächst (Abschnitt 4.1) wird berichtet, ob sich in der Datenstruktur übergeordnete Lernformen finden lassen. Danach werden die resultierenden Skalen bezüglich der Forschungsfragen analysiert (Abschnitt 4.2). 
Bestimmung übergeordneter Lernformen

Die Faktoranalyse weist sechs Faktoren mit total $58.86 \%$ aufgeklärter Varianz im Datensatz nach. Die Gütekriterien sind akzeptabel (Determinante $=0.017$; $K M O=0.683$ und Bartlett-Test: $p<0.001$; Anti-Image Korrelationen > 0.5).

In Abbildung 2 ist die resultierende rotierte Faktorlösung dargestellt. Die beiden ersten Faktoren lassen sich den traditionellen Unterrichtsformen (Erklären, Vorzeigen, Abfragen, Vorrechnen und Ganzklassenunterricht) sowie den innovativen Unterrichtsformen (Werkstattunterricht, Freiarbeit und Projektarbeit, Postenarbeit) zuordnen. Eigene Faktoren für die diskursiven Unterrichtsformen (Lehrgespräch, Diskussion und Präsentation) und für die Gruppenarbeit (Tandemarbeit und Gruppenarbeit) lassen sich nachweisen. Die Faktoranalyse weist weitere Faktoren für die Sozialform (Unterricht in Gruppen/ im Lerntandem), offenen Formen bzw. Formen mit Wablfreiheit (Wahl der Sozialform und Planarbeit) sowie Stillarbeit (Stillarbeit und Einzelarbeit) nach. Einzig das Item «Forschendes Lernen» lädt auf keinen der extrahierten Faktoren in bedeutendem Ausmass. Entsprechend wird das Item aus den weiteren Analysen ausgeschlossen.

Die beiden den Faktor Stillarbeit bildenden Items werden aufgrund ihrer inhaltlichen Nähe nicht überraschend demselben Faktor zugeordnet (vgl. dazu Problematik der gemeinsamen Analyse in Abschnitt 3.3) ${ }^{10}$. Es ist folglich möglich, dass der Faktor ein Relikt aufgrund der Oberflächenstruktur der Itemformulierung darstellt (ähnliches gilt für die Faktoren Gruppenarbeit und offenen Formen bzw. Formen mit Wahlfreiheit). Die Stillarbeit wird im Folgenden trotzdem als eigener Faktor behandelt, da sie einerseits einen gewichtigen Aspekt im Mathematikunterricht einnimmt und andererseits die den Faktor bildenden Items kaum einem anderen Faktor zugeordnet werden können (nur marginale Ladungen der Items auf den anderen Faktoren). Die beschriebene Faktorstruktur wird zudem beibehalten, da die getrennte Faktoranalyse für die Unterrichtsformen mit der beschriebenen Lösung korrespondiert: Die Faktoranalyse ergibt 5 Faktoren, denen die identischen Unterrichtsformen zugeordnet werden können. Der Faktor Gruppenarbeit fällt entsprechend weg. Ein Unterschied besteht in der Reihenfolge der Faktoren. Bei der getrennten Analyse erklären die innovativen Unterrichtsformen am meisten Varianz. Die Stillarbeit bildet einen eigenen Faktor. Auch bei der getrennten Analyse lässt sich das Forschende Lernen keinem Faktor zuordnen.

Die so gebildeten Skalen weisen, trotz der teilweise geringen Anzahl an Items, für den Untersuchungsgegenstand akzeptable Reliabilitätswerte auf (traditionelle Unterrichtsformen: Cronbach's $\alpha=0.685$, innovative Unterrichtsformen: $\alpha=0.697$, diskursive Unterrichtsformen: $\alpha=0.578$ ). Einzig die aus zwei Items bestehenden Skalen fallen bezüglich dem Cronbach's Alpha etwas ab, weisen aber hohe Korrelationen untereinander und eher mässige bis nicht vorhandene Korrelationen mit anderen Items auf (Gruppenarbeit: $r=0.446$, Stillarbeit: $r=0.358$ und offene Unterrichtsformen: $r=0.345$ ). 
Rotierte Komponentenmatrix ${ }^{a}$

\begin{tabular}{|c|c|c|c|c|c|c|}
\hline & \multicolumn{6}{|c|}{ Komponente } \\
\hline & 1 & 2 & 3 & 4 & 5 & 6 \\
\hline Vortrag_Erklärung & .621 & & & & -.341 & \\
\hline Vorzeigen & .665 & & & & & \\
\hline Abfragen & .663 & & & & & \\
\hline Vorrechnen lassen & .689 & & & & & \\
\hline Lehrgespräch & .420 & & .486 & -393 & & \\
\hline Diskussionen & & & .777 & & & \\
\hline $\begin{array}{l}\text { Präsentationen der } \\
\text { Lemenden }\end{array}$ & & & .779 & & & \\
\hline Stillarbeit & & & & & & .704 \\
\hline Postenarbeit & & .533 & & & .336 & \\
\hline Werkstattunterricht & & .757 & & & & \\
\hline Planarbeit & & & & & .710 & \\
\hline Freiarbeit & & .763 & & & & \\
\hline Projektunterricht & & .720 & & & & \\
\hline Forschendes Lernen & & & & .344 & & -434 \\
\hline Klassenarbeit & .594 & & & & & \\
\hline Tandemarbeit & & & & .746 & & \\
\hline Gruppenarbeit & & & & .731 & & \\
\hline Einzelarbeit & & & & & & .727 \\
\hline Wahl der Sozialform & & & & & .749 & \\
\hline
\end{tabular}

Extraktionsmethode: Analyse der Hauptkomponente.

Rotationsmethode: Varimax mit Kaiser-Normalisierung.

a. Rotation konvergierte in 9 Iterationen.

Abbildung 2: Rotierte Komponentenmatrix der Hauptachsen-Faktoranalyse. Ladungen kleiner als 0.3 werden in der Abbildung nicht angezeigt.

Einsatz von Lernformen im Mathematikunterricht Die Varianzanalyse ${ }^{11}$ weist eine signifikante Interaktion von Kanton und Schulstruktur nach $\left(p=0.038, \omega^{2}=0.016\right)$. Die Analyse der Mittelwerte (Abbildung 3) zeigt auf, dass im Kanton Graubünden in Mehrjahrgangsklassen öfter Traditionelle Unterrichtsformen angewendet als in Jahrgangsklassen, im Kanton Thurgau ist dies umgekehrt. Die relativ kleine Effektstärke und das Fehlen weiterer signifikanter Interaktionen und Haupteffekten zeigen allerdings auf, dass traditionelle Unterrichtsformen im Mathematikunterricht ähnlich oft zum Einsatz kommen.

Bezüglich der Innovativen Unterrichtsformen finden sich keine signifikanten Interaktions- und Haupteffekte, mit der Ausnahme der Variable Kanton $\left(p=0.049, \omega^{2}=0.014\right)$. Im Kanton Thurgau $(M=1.99)$ berichten die Lehrpersonen öfter vom Einsatz von innovativen Unterrichtsformen als im Kanton Graubünden $(M=1.81)$. Keine Unterschiede finden sich hingegen bei 


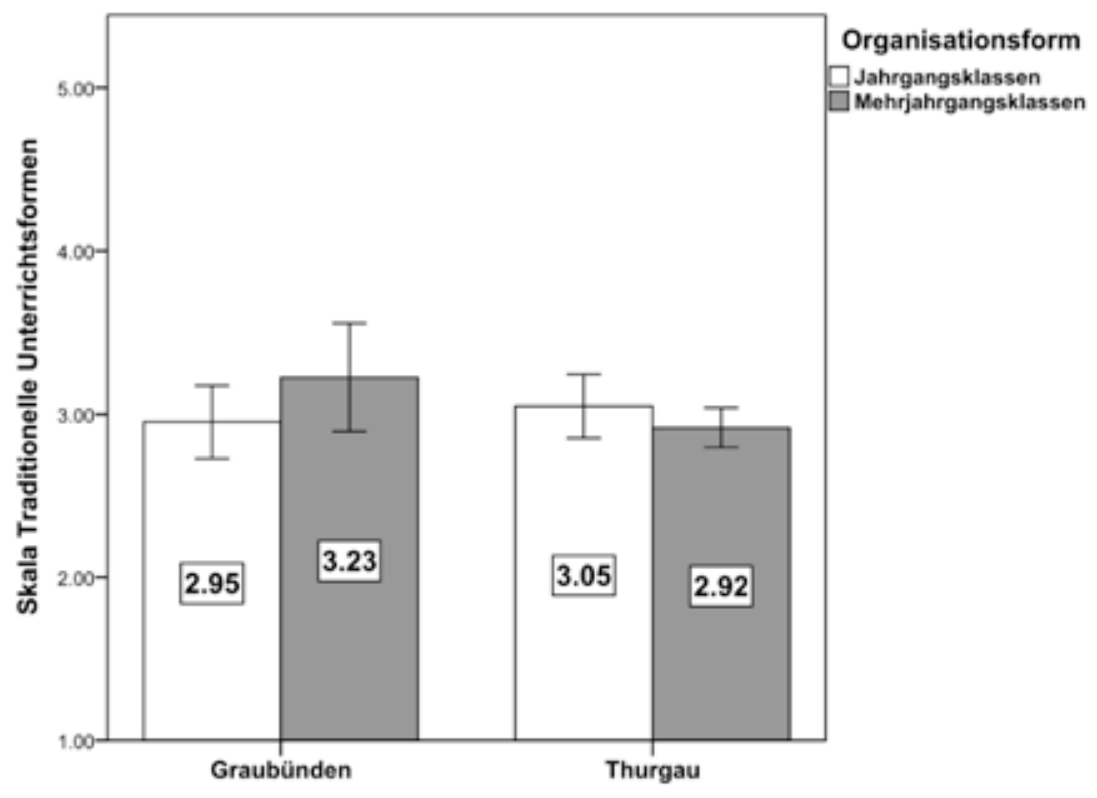

Fehlerbalken: $95 \% \mathrm{Cl}$

Jahrgangs- und Mehrjahrgangsklassen sowie bei Experten und Novizen.

Abbildung 3: Kantonsvergleich bezüglich der Schulstruktur: Traditionelle Unterrichtsformen (Mittelwerte).

Für den selbstberichteten Einsatz von Diskursiven Unterrichtsformen finden sich keine signifikanten Interaktions- und Haupteffekte, mit der Ausnahme der Dreifachinteraktion von Schulstruktur, Erfahrung und Kanton $(p=0.18$, $\left.\omega^{2}=0.022\right)$. Aufgrund der Schwierigkeit der Bestimmung von Dreifachinteraktionen und der geringen Effektstärke wird auf eine Interpretation verzichtet.

Bezüglich dem selbstberichteten Einsatz von Gruppenarbeiten findet sich eine signifikante Interaktion zwischen dem Kanton und der Erfahrung ( $p=0.003$, $\omega^{2}=0.036$ ). Die Analyse der Mittelwerte (Abbildung 4) zeigt auf, dass Gruppenarbeiten im Kanton Graubünden häufiger von Novizen als von Expertinnen eingesetzt werden. Im Kanton Thurgau ist dies, wenn auch knapp, umgekehrt. Es finden sich keine weiteren Interaktions- bzw. Haupteffekte.

Für den selbstberichteten Einsatz der Stillarbeit finden sich keine signifikanten Interaktions- und Haupteffekte. Die Stillarbeit wird unabhängig vom Kanton, Erfahrung und Schulstruktur in vergleichbarem Ausmass im Unterricht eingesetzt. 


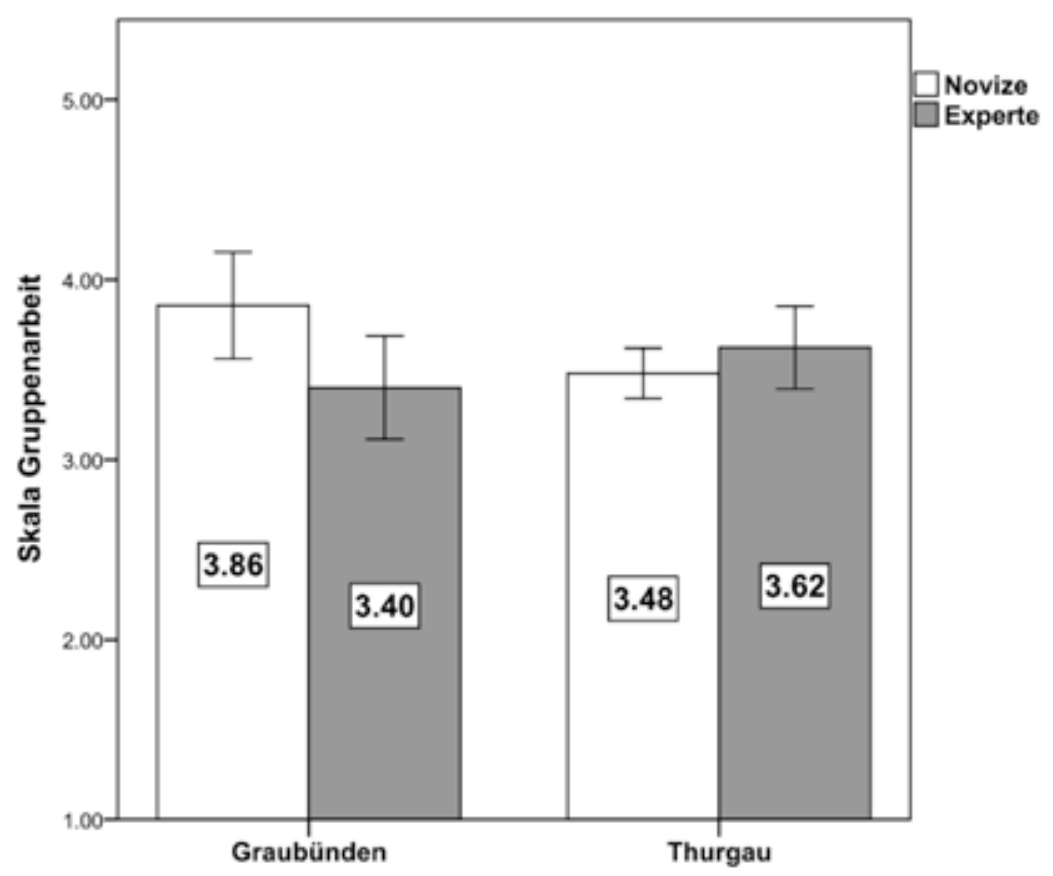

Fehlerbalken: $95 \% \mathrm{Cl}$

Abbildung 4: Kantonsvergleich bezüglich dem Expertenstatus: Gruppenarbeit (Mittelwerte).

Vergleichbar mit den Ergebnissen zu den innovativen Unterrichtsformen findet sich bei beim selbstberichteten Einsatz von offenen Formen ein signifikanter Unterschied zwischen den Kantonen, wobei die Lehrpersonen im Kanton Thurgau häufiger vom Einsatz von Formen mit Wahlfreiheit berichten $\left(M_{G R}=2.68, M_{T G}=3.14, p=0.017, \omega^{2}=0.023\right)$. Es finden sich keine weiteren signifikanten Haupteffekte bzw. Interaktionen.

Einzelmerkmale und Beantwortung der Fragestellungen Zusammengefasst zeigen die Daten der Befragung, dass der Mathematikunterricht sehr einheitlich gestaltet wird. Unabhängig von der Schulstruktur, des Expertenstatus oder des Kantons der befragten Lehrpersonen lassen sich wenige signifikante Unterschiede nachweisen. Die wenigen festgestellten signifikanten Unterschiede sind zudem klein, bzw. schwach. Die Fragestellungen (Abschnitt 2.3) lassen sich wie folgt beantworten: 
F1) Der Mathematikunterricht unterscheidet sich bezüglich der selbstberichteten Gestaltung bei Primarlehrpersonen in Mehrjahrgangsklassen nicht von derjenigen in Jahrgangsklassen.

F2) Es lassen sich keine Haupteffekte in der selbstberichteten Gestaltung des Mathematikunterrichts zwischen Experten und Novizen sowie zwischen Jahrgangs- und Mehrjahrgangsklassen finden.

F3) Feststellbar sind einzelne Kantonsunterschiede bezüglich des Einsatzes von offenen und innovativen Unterrichtsformen. Im Zusammenhang mit traditionellen Unterrichtsformen und Gruppenarbeiten zeigen sich Interaktionseffekte.

Ein Blick auf Detailanalysen (Abbildung 5) zeigt, welche Unterrichts- und Sozialformen wie häufig berichtet werden. Mathematikunterricht wird demnach stark von Stillarbeit sowie traditionellen Unterrichtsformen mit Vorzeigen, Klassenarbeit und Lehrgespräch geprägt, während innovative Unterrichtsformen wie Forschendes Lernen, Projekt- und Freiarbeit äusserst selten berichtet werden. Ebenfalls relativ selten berichtet werden diskursive Unterrichtsformen, während sich bei den offenen Unterrichtsformen eine relativ grosse Streuung zeigt. Werkstattunterricht wird relativ selten berichtet, während Posten- und Planarbeit einen festen Bestandteil des Mathematikunterrichts einzunehmen scheinen. Dies gilt auch für Gruppenarbeiten.

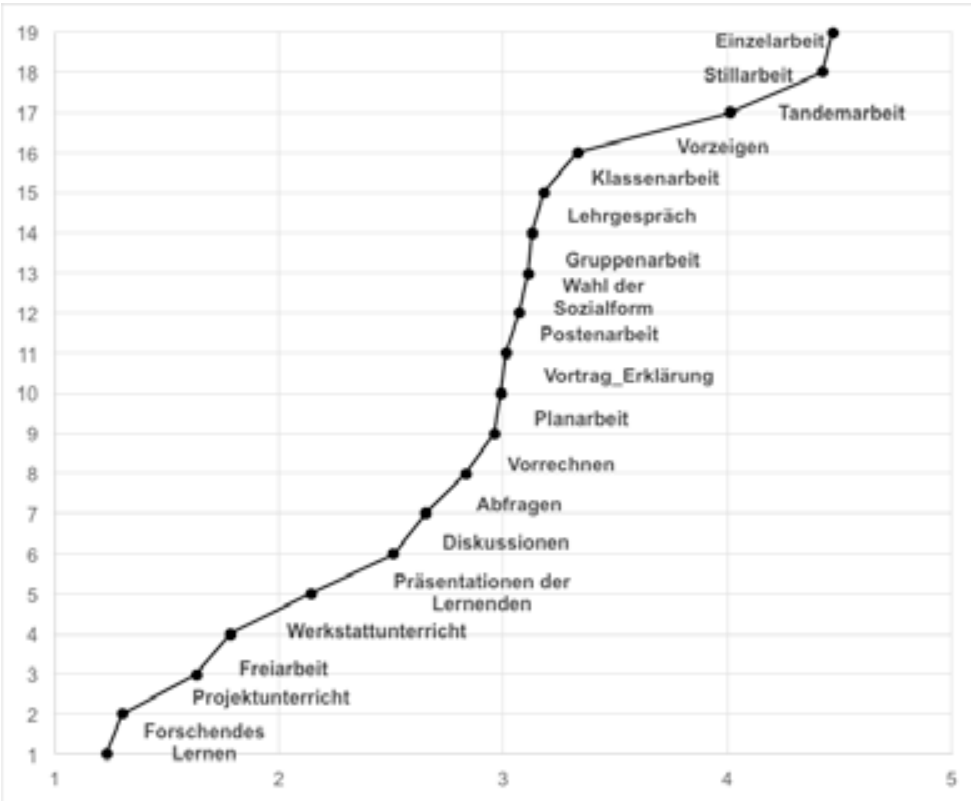

Abbildung 5: Mittelwerte der einzelnen Sozial- und Unterrichtsformen (1: weniger als 1 mal pro Monat; 2: 1-2 mal pro Monat; 3: 1 mal pro Woche; 4: 2-3 mal pro Woche; 5: (fast) jede Lektion). 


\section{Diskussion}

Haben schulstrukturelle Massnahmen wie das Implementieren von Mehrjahrgangsklassen anstelle von Jahrgangsklassen den erhofften Effekt, d.h. werden vermehrt erweiterte Lehr-/Lernformen im Mathematikunterricht umgesetzt? Die Ergebnisse zeigen, dass dies kaum der Fall ist. Dies stimmt auch überein mit bisher vorliegenden Befunden (Abschnitt 2.1). Es zeigen sich unabhängig der Schulstruktur ähnliche Gestaltungsmuster des Mathematikunterrichts für Jahrgangs- wie Mehrjahrgangsklassen, die zudem auch grossmehrheitlich unabhängig von der Berufserfahrung der Lehrpersonen und der Kantonszugehörigkeit sind. Der weitgehend fehlende Kantonsunterschied ist auch deshalb von Interesse, weil die beiden Kantone ihre Mehrjahrgangsklassen aufgrund unterschiedlicher Überlegungen führen (Abschnitt 1).

Generell ist Methodenvielfalt feststellbar, obwohl eine Dominanz von Stillund Gruppenarbeit vorliegt, während innovative sowie diskursive Unterrichtsformen deutlich seltener berichtet werden. Traditionelle sowie offene Unterrichtsformen hingegen werden regelmässig eingesetzt. Offene Unterrichtsformen mit einer grösseren Wahl der Lernenden werden im Kanton Thurgau häufiger berichtet als im Kanton Graubünden, während sich kein Unterschied zwischen Mehrjahrgangs- und Jahrgangsklassen zeigt. Gerade offene Formen wie Arbeitspläne und die Wahl der Sozialform zeigen, dass die Offenheit nicht inhaltlicher, sondern organisatorischer bzw. sozialer Art ist. Dies scheint im Thurgau stärker genutzt zu werden.

Dass Stillarbeit in allen Gruppen mit Abstand als am verbreitetsten berichtet wird und demgegenüber diskursive Unterrichtsformen selten eingesetzt werden, wirft im Hinblick auf den Aufbau von Problemlöse- und Argumentationskompetenzen Fragen auf. Denn diese Kompetenzen können nicht in Stillarbeit aufgebaut werden, sondern verlangen zwingend den Austausch mit einem Gegenüber. Es fragt sich deshalb, ob genügend Angebote zum Erwerb von diskursiv angelegten Kompetenzen geschaffen werden. Die starke Verbreitung von Gruppenarbeiten lässt keine inhaltliche Bestimmung zu. Hier müssten weitere Analysen folgen, die aufzeigen, für welche inhaltlichen Anforderungen Gruppenarbeiten genutzt werden (z. B. Matter, 2017).

Interessant sind die wenigen gefundenen Kantons- und Erfahrungsunterschiede: Wenn im Kanton Thurgau traditionelle Unterrichtsformen stärker in Jahrgangs- als in Mehrjahrgangsklassen eingesetzt werden, im Kanton Graubünden hingegen umgekehrt, so kann vermutet werden, dass der Umbau der Schulstruktur im Kanton Thurgau allenfalls einen leichten Effekt im Hinblick auf weniger traditionelle Unterrichtsformen hat. In die gleiche Richtung weist der Befund, wonach im Kanton Thurgau generell mehr innovative Unterrichtsformen berichtet werden als im Kanton Graubünden. Dass Gruppenarbeiten im Kanton Graubünden stärker von Novizinnen als von Experten genutzt werden, während dies im Kanton Thurgau umgekehrt ist, könnte mit der inhaltlichen 
Unbestimmtheit von Gruppenarbeiten zusammenhängen oder ev. darauf hindeuten, dass im Kanton Thurgau insbesondere von den erfahrenen Lehrpersonen tendenziell eher innovative Unterrichtsformen wie Posten-, Frei- und Projektarbeit gewählt werden anstelle von Gruppenarbeiten, während Gruppenarbeiten eher von Novizen eingesetzt werden, während im Kanton Graubünden die Novizen mit Gruppenarbeiten eine vermehrte Binnendifferenzierung zu realisieren versuchen. Mathematikunterricht folgt somit grossmehrheitlich von Schulstruktur, Erfahrung der Lehrperson und dem Schulkanton unabhängigen Gestaltungsmustern.

Von den Erwartungen (Abschnitt 2.2) kann keine bestätigt werden: Weder setzen Lehrpersonen in Mehrjahrgangsklassen vermehrt Gruppenarbeit noch mehr diskursive oder innovative Unterrichtsformen ein, als dies in Jahrgangsklassen der Fall ist. Allerdings werden im Kanton Thurgau weniger traditionelle Unterrichtsformen in Mehrjahrgangsklassen als in Jahrgangsklassen berichtet.

Mehrere Befunde erstaunen und verlangen nach intensivierter Forschung:

1) Die hohe Übereinstimmung von Expertinnen und Novizen in der selbstberichteten Gestaltung von Mathematikunterricht verlangt einerseits nach beobachtbaren, unabhängigen Einschätzungen der Qualität der Gestaltungsmuster (z. B. durch Videostudien) und andererseits nach der längsschnittlichen Erfassung von selbstberichteten Gestaltungsmerkmalen über die Berufslaufbahn hinweg.

2) Wenn sich die Gestaltung von Mathematikunterricht in Jahrgangs- und Mehrjahrgangsklassen bezüglich Unterrichtsformen so stark ähnelt, fragt sich, inwiefern die Altersmischung in Mehrjahrgangsklassen überhaupt genutzt wird oder ob diese Schulstruktur primär zu einer grösseren Organisation und damit höheren Belastung der Lehrperson führt, weil unterschiedliche Jahrgänge zum gleichen Zeitpunkt an verschiedenen Themen arbeiten. Lehrpersonen in Mehrjahrgangsklassen müssten demnach mehrere unterschiedliche inhaltliche Programme parallel bearbeiten, während sich Lehrpersonen in Jahrgangsklassen pro Zeitpunkt auf ein mathematisches Thema konzentrieren können. Dadurch wäre die zeitliche Belastung der Lehrpersonen in Mehrjahrgangsklassen gegenüber denjenigen aus Jahrgangsklassen höher. Weil den Lehrpersonen aus Mehrjahrgangsklassen aber nicht grundsätzlich mehr Vorbereitungszeit zur Verfügung steht als den anderen Lehrpersonen, müssen sie entweder zeitliche Ressourcen ausserhalb der Arbeitszeit nutzen oder sich mit kürzeren inhaltlichen Vorbereitungszeiten pro Thema begnügen, was zu geringerer Unterrichtsqualität führen könnte.

Die vorliegende Studie unterliegt verschiedenen Limitierungen: Der Datensatz mit den beiden unterschiedlich grossen Teilstichproben, die Kontrastierungen zulassen, ist relativ klein und nicht repräsentativ für die Gesamtpopulation der Primarlehrpersonen in einer Region. Zudem werden in der Fragebogenuntersuchung nur von den Lehrpersonen selbstberichtete Einschätzungen zur Häufigkeit bestimmter Unterrichts- und Sozialformen im Mathe- 
matikunterricht erhoben, nicht aber die tatsächliche Realisierung dieser in den Klassenzimmern. Die vorliegenden Daten lassen deshalb keine Aussagen über die Qualität der Umsetzung der Sozial- und Unterrichtsformen zu. Trotz allem zeigen die Befunde Tendenzen auf, und es kann angenommen werden, dass aufgrund der Stichprobenwahl und -gewinnung keine systematischen Verzerrungen vorliegen.

Im Rahmen der Studie ist es gelungen, sechs unterschiedliche Gestaltungsmuster des Mathematikunterrichts zu extrahieren. Diese Gestaltungsmuster können theoretisch bezüglich wesentlicher Dimensionen (Abschnitt 2.2) charakterisiert werden. Darüber hinaus lässt sich anhand der Gestaltungsmuster ein Bezug zum Kompetenzerwerb (D-EDK, 2014) herstellen und ausdifferenzieren, für welche Handlungsaspekte welches Gestaltungsmuster besonders effektiv sein dürfte. Die vorliegende Studie bildet u. E. eine wertvolle Basis, auf der weiter geforscht werden kann.

\section{Anmerkungen}

1 Z. B. bei Klassenzusammenlegungen aufgrund sinkender Schülerinnen- und Schülerzahlen.

2 Beobachtbar auch in den Kantonen Zürich, St. Gallen, Luzern sowie in grösseren Gemeinden des Kantons Appenzell Ausserrhoden.

3 Im Beitrag wird der deskriptive Begriff der «Mehrjahrgangsklasse» verwendet, der sich von der normativen Problematik, die mit dem Begriff «Altersdurchmischung» einhergeht, abgrenzt. Unter «Mehrjahrgangsklasse» wird das Zusammenlegen unterschiedlicher Jahrgänge in einem Schulzimmer verstanden. Über die Art der Unterrichtsgestaltung, sagt der Begriff nichts aus.

4 Im jahrgangsbezogenen Abteilungsunterricht werden die einzelnen Jahrgänge inhaltlich getrennt voneinander unterrichtet, was dazu führt, dass die Lehrperson mit einem Jahrgang arbeitet, während die anderen still für sich arbeiten.

5 Rossbach (2003) berichtet folgende vier Formen: 1) jahrgangsbezogener Abteilungsunterricht, 2) leistungshomogene Lerngruppen, 3) heterogene Lerngruppen und 4) Individualisierung der Lernprozesse.

6 Abzüglich der schon in der Zufallsstichprobe enthaltenen Lehrpersonen mit den Diplomjahren 2012/13 und $14(N=16)$.

7 Bei einer zweifaktoriellen Lösung bilden sich die Faktoren «traditionelle Unterrichtsformen» und «offene/innovative Unterrichtsformen». Die erhaltene Struktur bestätigt sich bei der Anwendung von nonparametrischen Klassifizierungsmethoden (nMDS).

8 Die Faktoranalyse weist aufgrund von einzelnen fehlenden Werten ein tieferes $N$ als 217 auf.

9 Die sechsfaktorielle Lösung wurde der zweifaktoriellen vorgezogen, da die Komplexität des Unterrichtsgeschehens in der Mathematik mit den zwei Faktoren traditionelle und offene/innovative Unterrichtsformen zu stark reduziert wird. Gerade wenn man die Dimensionen Steuerung und Sozialform/Diskursivität einbezieht, zeichnen sich mehr eigenständige Gestaltungsprofile ab. Auch eine Inspektion der Korrelationsmatrix lässt das Vorhandensein von distinkteren Formen der Unterrichtspraxis plausibel erscheinen.

10 Die Korrelation von $r=0.358$ spricht gegen die Annahme einer sehr starken Inhaltsabhängigkeit der beiden Items.

11 Die in Abschnitt 4.2 dargestellten Ergebnisse beziehen sich jeweils auf eine mehrfaktorielle Varianzanalyse mit den unabhängigen Variablen «Kanton» (TG/GR), «Schuls- 
truktur» (Mehrjahrgangsklasse/Jahrgangsklasse) und «Erfahrung» (Experte/Novize). Auf eine Anpassung der F-Werte aufgrund der ungleichen Gruppengrössen wurde verzichtet, da sich bei allen dargestellten Varianzanalysen homogene Varianzen nachweisen lassen (Levene-Test zwischen $p=0.175$ und $p=0.752$ ).

\section{Literatur}

Atteslander, P. (2003). Methoden der empirischen Sozialforschung. Berlin: de Gruyter. Bildungsdirektion Kanton Zürich. (2007). Projekt Neugestaltung des 9. Schuljahres. Planungshilfe für Pilotschulen. Zürich: Bildungsdirektion Kanton Zürich.

Brophy, J. E. \& Good, T. L. (1986). Teacher behavior and student achievement. In M. C. Wittrrock (Ed.), Handbook of Research on Teaching (3th ed., pp. 328-375). London: Macmillan.

Brunner, E. (2017). Mathematikunterricht in Mehrjahrgangsklassen der Primarschule: Eine Deskription entlang verschiedener Gestaltungselemente und Einschätzungen der Lehrpersonen. Journal für Mathematik-Didaktik, 38(1), 57-91.

Carle, U. \& Berthold, B. (2004). Schuleingangsphase entwickeln. Leistungen fördern. Wie 15 staatliche Grundschulen in Thüringen die flexible, jahrgangsgemischte und integrative Schuleingangsphase einrichten. Baltmannsweiler, Deutschland: Schneider Verlag Hohengehren.

D-EDK. (2014). Lehrplan 21. Mathematik. Bern: Projekt Lehrplan 21.

Duss, U. \& Gallin, P. (2015). Ist alles zählbar? Erschliessung des Zahlenraums mit einer altersdurchmischten Lerngruppe der Unterstufe. Schweizerische Zeitschrift für Heilpädagogik, 21(4), 35-42.

Fend, H. (1998). Qualität im Bildungswesen: Schulforschung zu Systembedingungen, Schulprofilen und Lehrerleistung. Weinheim, Deutschland: Juventa.

Feuser, G. (2013). Die «Kooperation am Gemeinsamen Gegenstand». Behinderte Menschen, Zeitschrift für gemeinsames Leben, Lernen und Arbeiten, 2, 17-35.

Field, A. (2014). Discovering statistics using IBM SPSS Statistics (4th ed.). Los Angeles, CA: Sage.

Gallin, P. (2015). Dialogischer Mathematikunterricht auf jedem Niveau. Schweizerische Zeitschrift für Heilpädagogik, 21(4), 20-26.

Greeno, J. G. (2006). Authoritative, accountable positioning and connected, general knowing: Progressive theme in understanding transfer. The Journal of the Learning Sciences, 15(4), 537-547.

Grittner, F., Hartinger, A. \& Rehle, C. (2013). Wer profitiert beim jahrgangsgemischten Lernen? Zeitschrift für Grundschulforschung, 6(1), 102-113.

Hattie, J. (2009). Visible learning. A synthesis of over 800 meta-analyses relating to achievement. London: Routledge.

Helmke, A. (2012). Unterrichtsqualität und Lehrerprofessionalität. Diagnose, Evaluation und Verbesserung des Unterrichts (4. Aufl.). Seelze, Deutschland: Kallmeyer.

Huberty, C. J. \& Morris, J. D. (1989). Multivariate analysis versus multiple univariate analyses. Psychological Bulletin, 105(2), 302-308.

Hyry-Beihammer, E. K. \& Hascher, T. (2015). Multi-grade teaching practices in Austrian and Finnish primary schools. International Journal of Educational Research, 2015(74), 104-113.

Kadivar, P., Navabi Nejad, S. \& Madadi Emamzade, Z. (2005). Effectiveness of multi-grade classes: Cooperative learning as a key element of success. Proceedings of world adademy of science, engineering and technology, 8, 169-172.

Kucharz, D. \& Wagener, M. (2009). Jahrgangsübergreifendes Lernen. Baltmannsweiler, Deutschland: Schneider Verlag Hohengehren. 
Laging, R. (2003). Altersmischung - eine pädagogische Chance. In R. Laging (Hrsg.), Altersgemischtes Lernen in der Schule (S. 1-29). Baltmannsweiler, Deutschland: Schneider Verlag Hohengehren.

Largo, R. H. (2013). Kinderjahre: die Individualität des Kindes als erzieherische Herausforderung (25. Aufl). München: Piper.

Matter, B. (2017). Lernen in heterogenen Lerngruppen. Erprobung und Evaluation eines Konzepts für den jahrgangsgemischten Mathematikunterricht. Wiesbaden, Deutschland: Springer Spektrum.

Monn, X. (2011). AdL - Anmerkungen zu einem polarisierenden Begriff. Schulblatt des Kantons Thurgau, 6, 9-11.

Mulryan-Kyne, C. M. (2004). Teaching and learning in multigrade classrooms: What the teachers say. Irish Journal of Education, 35, 5-19.

Nührenbörger, M. (2009). Interaktive Konstruktionen mathematischen Wissens - Epistemologische Analysen zum Diskurs von Kindern im jahrgangsgemischten Anfangsunterricht. Journal für Mathematikdidaktik, 30(2), 147-172.

Pauli, C. \& Reusser, K. (2001). Doku: Lehrerbefragung im schweizerischen Videoprojekt. Unveröffentlichte Projektunterlage, Zürich: Universität.

Raggl, A. (2011). Altersgemischter Unterricht in kleinen Schulen im alpinen Raum. In R. Müller, A. Keller, U. Kerle \& E. Steiner (Hrsg.), Schule im alpinen Raum (S. 231-305). Innsbruck: Studienverlag.

Raggl, A., Smit, R. \& Kerle, U. (2015). Kleine Schulen im ländlich-alpinen Raum. Innsbruck: StudienVerlag.

Rakoczy, K., Buff, A. \& Lipowsky, F. (2005). Befragungsinstrumente. In E. Klieme, C. Pauli $\&$ K. Reusser (Hrsg.), Dokumentation der Erhebungs- und Auswertungsinstrumente zur schweizerisch-deutschen Videostudie «Unterrichtsqualität, Lernverhalten und mathematisches Verständnis» (Bd. 1). Frankfurt: DIPF.

Reusser, K. (2005). Problemorientiertes Lernen - Tiefenstruktur, Gestaltungsformen, Wirkung. Beiträge zur Lehrerbildung, 23(2), 159-182.

Reusser, K. \& Pauli, C. (2010). Unterrichtsgestaltung und Unterrichtsqualität - Ergebnisse einer internationalen und schweizerischen Videostudie zum Mathematikunterricht: Einleitung und Überblick. In K. Reusser, C. Pauli \& M. Waldis (Hrsg.), Unterrichtsgestaltung und Unterrichtsqualität - Ergebnisse einer internationalen und schweizerischen Videostudie zum Mathematikunterricht (S. 9-32). Münster: Waxmann.

Rossbach, H.-G. (2003). Empirische Vergleichsuntersuchungen zu den Auswirkungen von jahrgangsheterogenen und jahrgangshomogenen Klassen. In R. Laging (Hrsg.), Altersgemischtes Lernen in der Schule (2. korr. Aufl., S. 80-91). Hohengehren: Schneider.

Schülke, C. (2013). Mathematische Reflexion in der Interaktion von Grundschulkindern: theoretische Grundlegung und empirisch-interpretative Evaluation. Münster: Waxmann.

Thurn, S. (2010). Leben, lernen, leisten in jahrgangsübergreifenden Gruppen. In A. Buholzer \& A. Kummer Wyss (Hrsg.), Alle gleich - alle unterschiedlich! Zum Umgang mit Heterogenität in der Schule (S. 28-39). Zug: Klett \& Balmer.

Trachsler, E. (2008). Altersdurchmischte Klassen in der Thurgauer Volksschule Eine eplorative Studie zu einer vielversprechenden Schulform zwischen Tradition und Innovation. Kreuzlingen: Pädagogische Hochschule.

Wagener, M. (2014a). Welche Erfahrungen machen Lehrerinnen und Lehrer. Die Grundschulzeitschrift, (274), 46-47.

Wagener, M. (2014b). Potenziale der Jahrgangsmischung. Die Grundschulzeitschrift, 274), 29-33.

Zumwald, B. (2012). Altersmischung und Aufgabenstellungen in der jahrgangsgemischten Eingangsstufe in der Schweiz. In U. Carle \& J. Kosinar (Hrsg.), Aufgabenqualität in Kindergarten und Grundschule - Grundlagen und Praxisbeispiele (S. 53-66). Baltmannsweiler: Schneider Verlag Hohengehren. 
Schlagworte: Mathematikunterricht, Unterrichtsformen, Jahrgangsklassen, Mehrjahrgangsklassen, Altersdurchmischung

\section{Exemple de conception de cours de mathématiques dans les classes à niveau unique et à niveaux multiples - comparatif entre 2 cantons}

\section{Résumé}

La présente étude a été menée dans les cantons de Thurgovie et des Grisons afin d'étudier dans quelle mesure le recours à certaines formes sociales d'enseignement dans le cadre des cours de mathématiques faisait une différence dans les classes à niveau unique et à niveaux multiples à l'école primaire. L'enquête a été menée auprès d'enseignants expérimentés et en début de carrière. Les données recueillies permettent de faire des comparaisons entre les cantons, les experts et les novices et les classes à niveau unique ou à niveaux multiples. Les résultats montrent que la conception de cours de mathématiques présente plus de points communs que de disparités. Cela a également permis d'extraire six critères qui peuvent être interprétés comme autant de profils de conception.

Mots-clés: Cours de mathématiques, types de cours, classes à niveau unique, classes à niveaux multiples, mélange des âges

\section{Modelli organizzativi di didattica della matematica in pluriclassi e classi per età - due cantoni a confronto}

\section{Riassunto}

La presente ricerca ha esaminato nei cantoni Turgovia e Grigioni in che misura l'applicazione (descritta dai docenti) di specifiche forme didattiche e sociali si differenzia nell'insegnamento della matematica in classi per età e in pluriclassi della scuola elementare. Le interviste hanno coinvolto in parte docenti esperti e in parte docenti all'inizio del loro percorso professionale. L'insieme dei dati consente confronti tra cantoni, tra docenti esperti e novizi e tra pluriclassi e classi per età. I risultati indicano come dal punto di vista dell'organizzazione (autodescritta) dell'insegnamento della matematica risultino decisamente più aspetti comuni che differenti. Si sono inoltre potuti estrarre sei fattori che possono essere interpretati in quanto distinti profili organizzativi.

Parole chiave: didattica della matematica, forme didattiche, classi per età, pluriclassi, classi di età eterogenee 


\section{Organisation patterns of mathematics teaching in single- age and multi-age classrooms - a comparison between two Swiss cantons}

\section{Summary}

Focusing on mathematics education at primary schools in the Swiss cantons of Thurgau and Graubünden, our study aimed to find out how the self-reported use of particular teaching methods and forms of social organisation differs between single-age classes and multi-age classes. The survey was conducted among experienced teachers and teachers at the beginning of their professional career. The database thus permits comparisons between two cantons, experts and novices as well as between single-age and multi-age classes. Our findings indicate that the groups reported far more commonalities than differences with respect to how they organise mathematics lessons. Furthermore, we were able to extract six factors which can be interpreted as different organisation patterns (i.e. profiles).

Keywords: Mathematics teaching, organisation patterns, single-age classrooms, multi-grade classrooms, age-mixed learning 